Hydrobiologia 192: 279, 1990.

H. L. Golterman (ed.). Sediment-Water Interaction.

(C) 1990 Kluwer Academic Publishers. Printed in Belgium.

\title{
Erratum
}

\section{Nitrogen dynamics in a eutrophic lake sediment}

K. R. Reddy, R. E. Jessup \& P. S. C. Rao

University of Florida, Institute of Food and Agricultural Sciences, Soil Science Department, Gainesville, FL 32611, USA

Hydrobiologia 159: 177-188, 1988.

Equation (1) on page 178 should read

$$
\ell^{w} \frac{\partial \mathrm{N}_{4}}{\partial t}=\ell^{w} k_{4}^{w} \mathrm{~N}_{\mathrm{org}}-\ell^{w} k_{3}^{w} \mathrm{~N}_{4}+D_{4}^{x} \theta^{x}\left(\frac{\partial \mathrm{N}_{4}}{\partial z}\right)_{z=0}-V_{4}
$$

